\title{
The TLR9 agonist MGN1703 triggers a potent type I interferon response in the sigmoid colon
}

\author{
AR Krarup ${ }^{1,17}$, M Abdel-Mohsen ${ }^{2,3,4,17}$, MH Schleimann ${ }^{1,17}$, L Vibholm $^{1,5}$, PA Engen ${ }^{6}$, A Dige $^{7}$, B Wittig $^{8}$, \\ M Schmidt ${ }^{9}$, SJ Green ${ }^{10,11}$, A Naqib ${ }^{11}$, A Keshavarzian ${ }^{6}$, X Deng ${ }^{2}$, R Olesen ${ }^{1}$, AM Petersen ${ }^{12,13}$, \\ T Benfield $^{14}$, L Østergaard ${ }^{1,5}$, TA Rasmussen ${ }^{1}$, J Agnholt ${ }^{5,7}$, JR Nyengaard ${ }^{5,15}$, A Landay ${ }^{16}$, \\ OS Søgaard ${ }^{1,5}$, SK Pillai ${ }^{2,3}$, M Tolstrup $^{1,5}$ and PW Denton ${ }^{1,5}$
}

Toll-like receptor 9 (TLR9) agonists are being developed for treatment of colorectal and other cancers, yet the impact of these drugs on human intestines remains unknown. This, together with the fact that there are additional potential indications for TLR9 agonist therapy (e.g., autoimmune and infectious diseases), led us to investigate the impact of MGN1703 (Lefitolimod) on intestinal homeostasis and viral persistence in HIV-positive individuals. Colonic sigmoid biopsies were collected (baseline and week four) from 11 HIV + individuals on suppressive antiretroviral therapy, who received MGN1703 (60 mg s.c.) twice weekly for 4 weeks in a single-arm, phase 1b/2a study. Within sigmoid mucosa, global transcriptomic analyses revealed 248 modulated genes (false discovery rate $<0.05$ ) including many type I interferon (IFN)-stimulated genes. MGN1703 increased the frequencies of cells exhibiting MX1 $(P=0.001)$ and ISG15 $(P=0.014)$ protein expression. No changes were observed in neutrophil infiltration (myeloperoxidase; $P=0.97$ ).

No systematic effect on fecal microbiota structure was observed (analysis of similarity Global $R=-0.105 ; P=0.929$ ). TLR9 expression at baseline was inversely proportional to the change in integrated HIV DNA during MGN1703 treatment $(P=0.020)$. In conclusion, MGN1703 induced a potent type I IFN response, without a concomitant general inflammatory response, in the intestines.

\section{INTRODUCTION}

The intracellular innate immune surveillance molecule Tolllike receptor 9 (TLR9) is a pattern recognition receptor found in plasmacytoid dendritic cells, B cells, and in some mucosal epithelial cells. ${ }^{1-4}$ DNA containing non-methylated cytosineguanine sequence motifs (CG- or CpG-motifs) bind to and activate TLR9 in either of two types of endosomes, depending on the structural context and CG-motif sequence environment. In one type of endosome, TLR9 signal transduction is via activation of nuclear factor- $\mathrm{\kappa B}$, which preferentially leads to transcription of proinflammatory cytokine genes. In the other endosome type, signal transduction is through interferon (IFN) regulatory factor 7 , which preferentially drives transcription of type I IFN genes. ${ }^{5,6}$ Paracrine and autocrine cytokine signaling then leads to activation of innate and adaptive immune responses. ${ }^{7,8}$ To clinically utilize TLR9's vital immune surveillance activity, families of synthetic DNA molecules were developed as TLR9 ligands (agonists and antagonists). TLR9 agonists have been and are being tested as vaccine adjuvants and as therapeutics for cancers-including colorectal

${ }^{1}$ Department of Infectious Diseases, Aarhus University Hospital, Aarhus, Denmark. ${ }^{2}$ Blood Systems Research Institute, San Francisco, California, USA. ${ }^{3}$ University of California, San Francisco, California, USA. ${ }^{4}$ The Wistar Institute, Philadelphia, Pennsylvania, USA. ${ }^{5}$ Department of Clinical Medicine, Aarhus University, Aarhus, Denmark. ${ }^{6}$ Division of Digestive Diseases and Nutrition, Department of Internal Medicine, Rush University Medical Center, Chicago, Illinois, USA. ${ }^{7}$ Department of Hepatology and Gastroenterology, Aarhus University Hospital, Aarhus, Denmark. ${ }^{8}$ Foundation Institute Molecular Biology and Bioinformatics, Freie Universitaet Berlin, Berlin, Germany. ${ }^{9}$ Mologen AG, Berlin, Germany. ${ }^{10}$ Department of Biological Sciences, University of Illinois at Chicago, Chicago, Illinois, USA. ${ }^{11}$ DNA Services Facility, Research Resources Center, University of Illinois at Chicago, Chicago, Illinois, USA. ${ }^{12}$ Department of Microbiology, Hvidovre Hospital, University of Copenhagen, Hvidovre, Denmark. ${ }^{13}$ Department of Gastroenterology, Hvidovre Hospital, University of Copenhagen, Copenhagen, Denmark. ${ }^{14}$ Department of Infectious Diseases, Hvidovre Hospital, Hvidovre, Denmark. ${ }^{15}$ Core Centre for Molecular Morphology, Section for Stereology and Electron Microscopy Laboratory, Centre for Stochastic Geometry and Advanced Bioimaging, Aarhus University Hospital, Aarhus, Denmark and ${ }^{16}$ Department of Immunology/Microbiology, Rush University Medical Center, Chicago, Illinois, USA. Correspondence: PW Denton (pade@clin.au.dk)

${ }^{17}$ These authors contributed equally to this work. 
carcinoma, infectious diseases, inflammatory bowel diseases, asthmas and allergies (e.g., ClinicalTrials.gov ID: NCT02077 868, NCT02200081, NCT02254772, and NCT02668770). ${ }^{9-14}$ However, the effects of different TLR9 agonists within the intestines have only been described in mice, sheep, and fish, ${ }^{15-17}$ whereas the physiological effects of any TLR9 agonist in human intestines were essentially unknown before this study.

In the context of inflammatory bowel diseases, TLR9 agonists could have a role in modulating aberrant immune responses that lead to inflammation, e.g., by inducing tolerance towards commensal bacteria. ${ }^{9-12}$ In the context of infectious diseases, TLR9 agonists may enhance immune surveillance activities that facilitate reductions in pathogen burden. ${ }^{18}$ Specifically, in the case of HIV eradication, therapeutic use of TLR9 agonists will likely be undertaken in a combination strategy within HIV-positive individuals on suppressive antiretroviral therapy (ART). Therefore, we enrolled such persons and performed a comprehensive assessment of the impact on intestinal homeostasis and inflammation by the TLR9 agonist and immune surveillance reactivator MGN1703 (lefitolimod).

MGN1703 is synthetic TLR9 agonist of the double-stem loop immunomodulators or dSLIM family where multiple CG-motifs are embedded into a stable, covalently closed double stem-loop DNA molecule harboring only natural phosphodiester bonds between nucleotides. ${ }^{19-21}$ MGN1703's pre-clinically determined efficacy parameters are maintained in clinical studies without dose-limiting toxicities ${ }^{19-21}$ and this drug is currently in phase III testing for treating metastatic colorectal cancer. ${ }^{21}$ The clinical proof-of-concept for MGN1703 was achieved in a clinical phase II study evaluating switch-maintenance treatment following standard chemotherapy of patients with metastatic colorectal carcinoma. ${ }^{21,22}$

Currently, there is a renewed interest in developing effective and accessible therapies that can lead to eradication of HIV infection or that facilitate sustained viral remission in the absence of ART. ${ }^{23}$ The main barrier to HIV eradication is the ability of the virus to establish latent infection in long-lived $\mathrm{CD}^{+}{ }^{+} \mathrm{T}$ cells that persist in the body despite ART. ${ }^{24}$ These latently infected cells are the likely source of plasma viral rebound after interruption of ART and there is compelling evidence that the intestines are a major reservoir for HIV persistence. $^{25,26}$ To eradicate persistent HIV, robust immune surveillance is required. ${ }^{27} \mathrm{We}$ previously showed (ex vivo and in vivo) that the cells of HIV-positive individuals responded appropriately to TLR9 agonist stimulation. ${ }^{18,28,29}$ Thus, TLR9 agonist-mediated enhancement of the activation status and killing capacity of $\mathrm{CD}^{+} \mathrm{T}$ and natural killer (NK) cells may augment the reduction or elimination of persistent viruses.

In our preclinical evaluation of MGN1703 ex vivo using peripheral blood mononuclear cells isolated from HIV infected individuals, we found that MGN1703 incubation results in NK cell activation and increased NK cell inhibition of HIV spreading in a culture of autologous $\mathrm{CD} 4+\mathrm{T}$ cells. ${ }^{18}$ As we examined the systemic effects evaluating MGN1703 effects in our clinical trial, we found that treatment in HIV infected individuals has a dual potential in HIV eradication efforts as MGN1703 increased HIV-1 transcription and enhanced cytotoxic NK cell activation in vivo. ${ }^{30}$ Here we report a sub-study of this trial where we define MGN1703-treatment outcomes related to: (i) TLR9 agonist-induced intestinal immune activity; (ii) maintenance of intestinal homeostasis during TLR9 agonist treatment; (iii) stability of the gut microbiota composition during TLR9 agonist treatment; and (iv) TLR9 agonist-associated changes in HIV persistence in the intestine.

\section{RESULTS \\ MGN1703 induced a potent type I IFN response in the intestines}

The clinical trial design is depicted in (Figure 1a). Participants received subcutaneous injections of MGN1703 (60 mg) two times per week for 4 weeks, while maintaining ART. We previously reported that MGN1703 dosing led to activation of peripheral blood plasmacytoid dendritic cell, cytotoxic NK cells, and CD8 $+\mathrm{T}$ cells, as well as substantial increases in plasma IFN- $\alpha 2$ levels in this cohort. ${ }^{30}$ For the current study, endoscopic collection of colonic sigmoid biopsies were performed at baseline and during the last week of MGN1703 administration. We used quantitative immunohistochemistry (IHC) on intestinal biopsy tissues to detect the canonical type I IFN-induced proteins MX1 and ISG15. ${ }^{31}$ We observed an increase in the numbers of MX1-positive cell profiles from baseline to the fourth week of treatment (Figure 1b) in both the intestinal lamina propria $(P=0.001)$ (Figure 1c) and epithelium $(P=0.003)$ (Figure 1d). The number of ISG15positive cell profiles in lamina propria also increased during MGN1703 treatment $(P=0.014)$ (Figure 1e), whereas no significant changes in the number of ISG15-producing cell profiles in the epithelial region were observed (Figure 1f). These protein-detection observations were consistent with the global transcriptomic analyses performed using RNA sequencing (RNASeq) on intestinal tissue mononuclear cells isolated from these individuals. We observed hierarchical clustering of samples in a treatment-dependent manner (Slatkin-Maddison $P=0.0167$; Figure 2a) and segregation of the response was also observed with principal component analyses (Figure $\mathbf{2 b}$ and Supplementary Figure $\mathbf{1}$ online). These findings indicate that there was a potent, dominant effect of MGN1703 administration on the intestinal transcriptome that supersedes inter-individual variability in gene expression. There were 248 genes whose expression was significantly modulated by MGN1703 treatment (fold change $>2$; false discovery rate $<0.05$ ) (Figure $2 c$ and Supplementary Table S1 online). In addition to upregulating MX1 and ISG15 expression, MGN1703 treatment led to significant increases in the expression levels of multiple additional IFN-stimulated genes (e.g., IFI6, OAS, and IFIT1), antiviral genes specifically associated with the control of HIV-1 in vivo (IFITM1, MX2, and TRIM22), as well as IFN pathway signaling molecules (e.g., STAT1 and IRF7) (Figures 2c and 3a). 


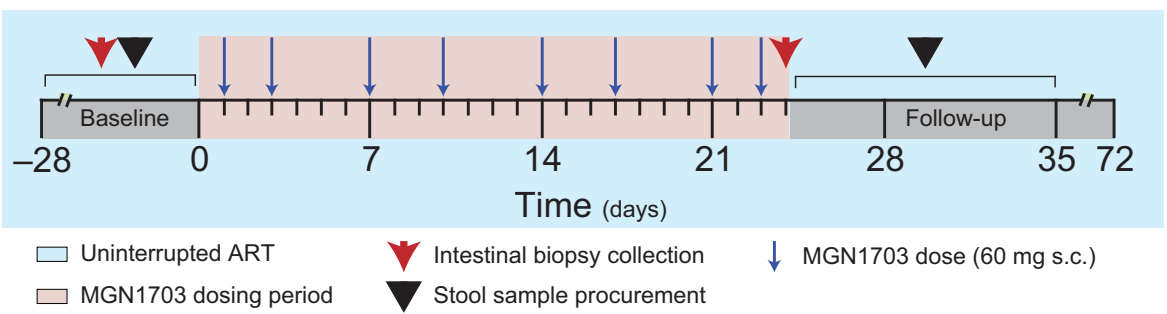

b

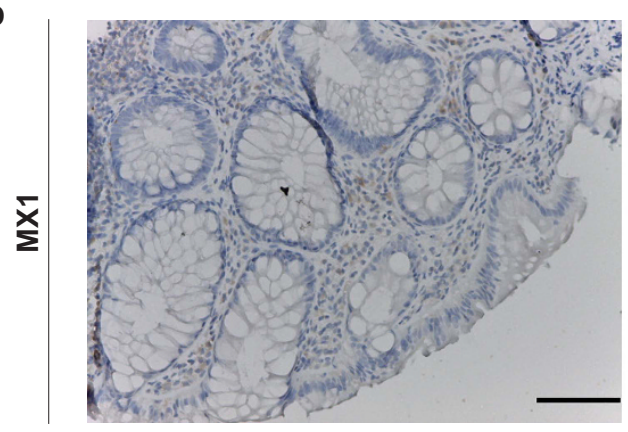

Baseline

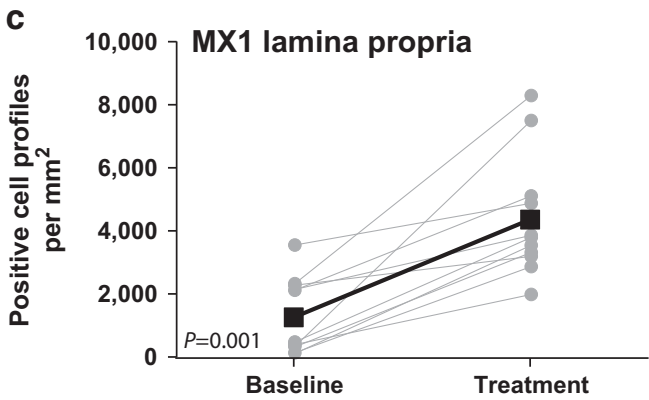

e

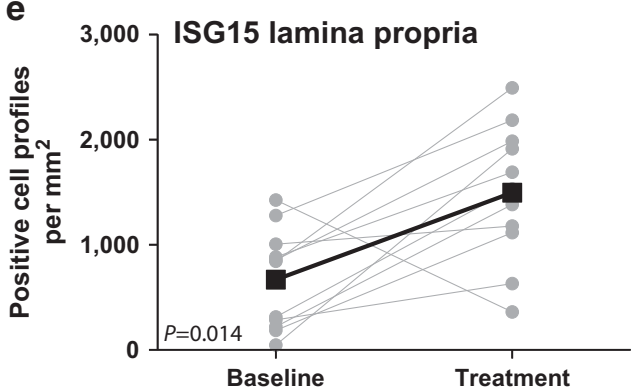

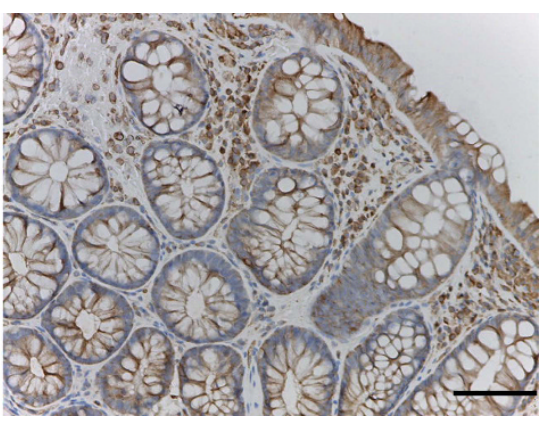

Treatment
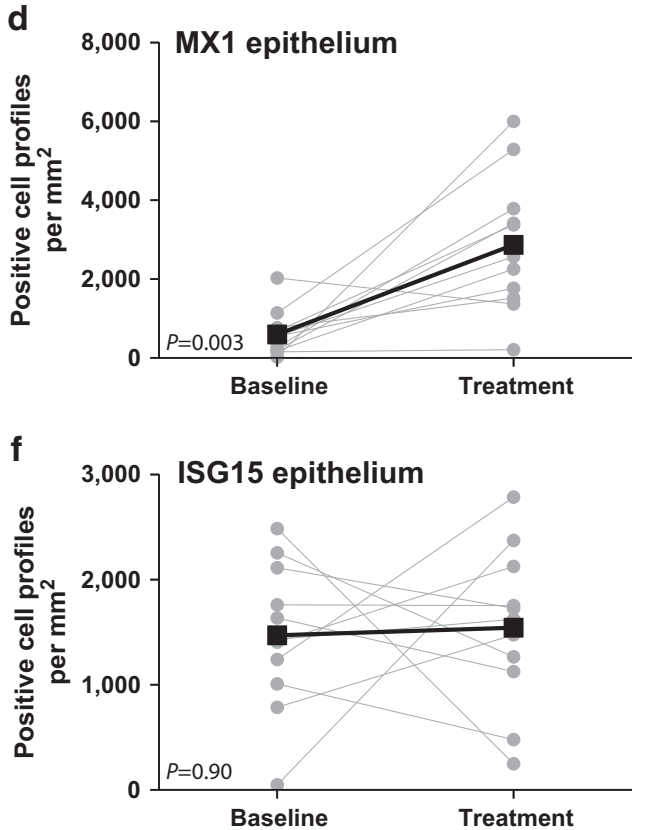

Figure 1 MGN1703 induced an increase in MX1 and ISG15-positive cell profiles in the sigmoid colon. (a) The timing of MGN1703 dosing and sample collection are illustrated. (b) Representative images for MX1 immunohistochemistry (IHC) (brown staining) from biopsies collected prior to dosing (baseline) and during the fourth dosing week (treatment) are presented. Scale bars, $100 \mu \mathrm{m}$. (c-f) Plots show the number of cell profiles expressing MX1 (c,d) or ISG15 (e,f) at baseline and during MGN1703 treatment ( $n=11$ in all graphs). Gray circles indicate results for each individual. Paired data are connected with gray lines. Black squares represent the mean value for all individuals at the indicated time point. Black lines connect longitudinal mean data points. (c,e) The numbers of positive cell profiles per $\mathrm{mm}^{2}$ within the lamina propria regions are illustrated. (d,f) The numbers of positive cell profiles per $\mathrm{mm}^{2}$ within the epithelial regions are illustrated. Statistics: Wilcoxon matched-pairs signed-rank test.

MGN1703 treatment was not associated with an induction of type II IFN responses or markers of inflammation in the intestines

Secondary effects of IFN- $\alpha$ production can include the activation of a range of effector cells (e.g., IFN- $\alpha$ can activate NK cells to secrete type II IFN (i.e., IFN- $\gamma$ )). ${ }^{32}$ In our study, significant modulation of IFN- $\gamma$ and type II IFN response genes [(e.g., RELA (nuclear factor- $\kappa \mathrm{B}$ p65), IL6, CXCL8 (interleukin
(IL)-8), and TNF (tumor necrosis factor- $\alpha$ )) was not detected in RNASeq analyses of intestinal mononuclear cells (Figure 3a). Consistent with no change in the levels of IFN- $\gamma$ mRNA in the intestinal tissue mononuclear cells, we did not detect significant changes in the numbers of cell profiles positive for the IFN- $\gamma$ induced protein CXCL10 using IHC in either the intestinal lamina propria (Figure 3b) or epithelium (Figure 3c). Nor was MGN1703 treatment associated with changes in intestinal 
a
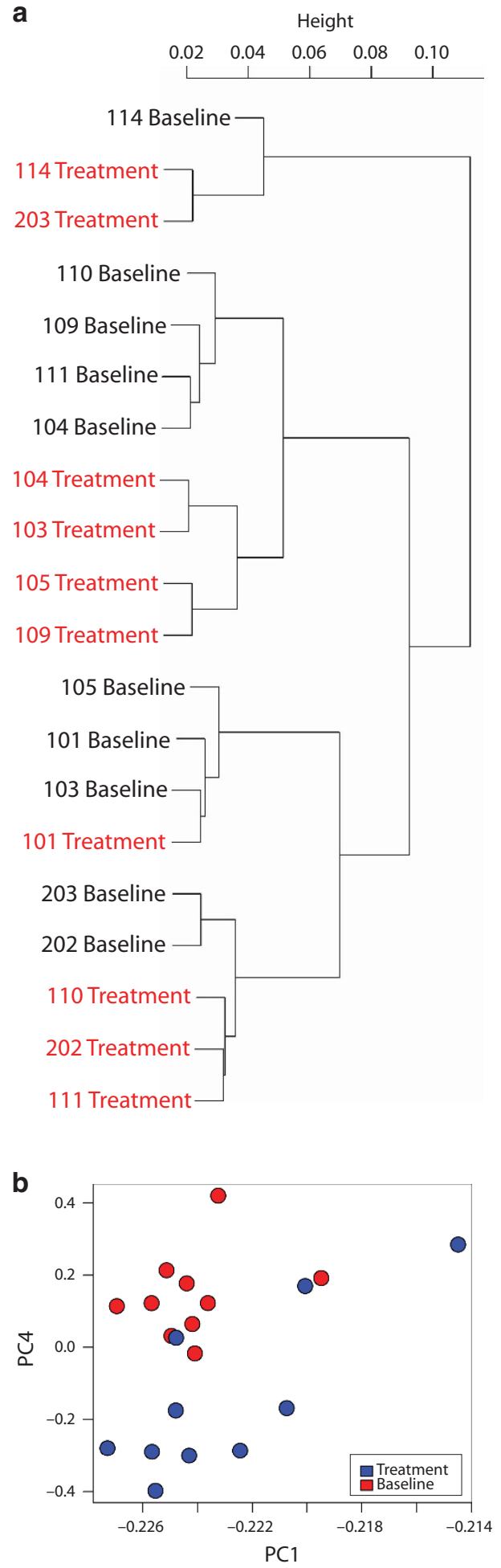

C

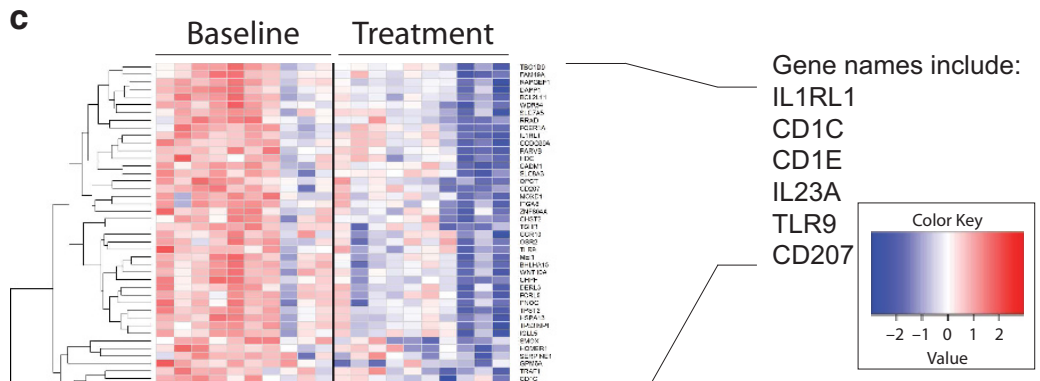

Gene names in order for this region:

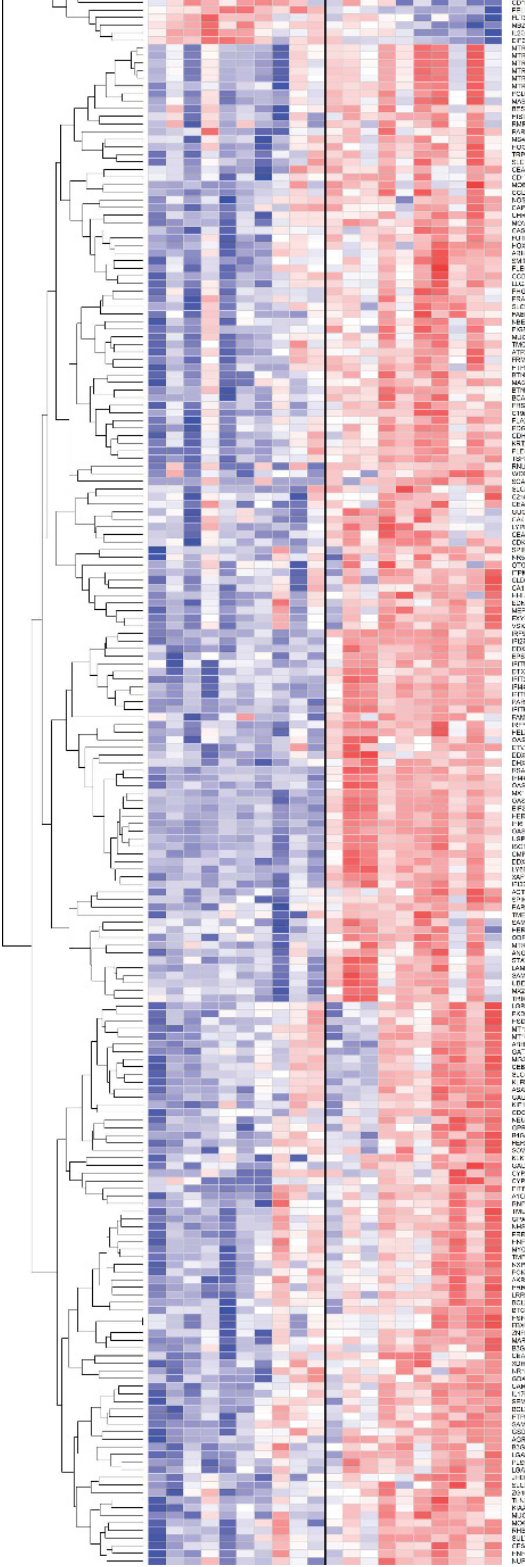

DDX60

EPSTI1

IFIT5

DTX3L

IFIT3

IFI44

IFIT1

PARP9

IFITM1

FAM177B

IRF7

HELZ2

OASL

ETV7

DDX60L

DHX58

RSAD2

IFI44L

OAS3

MX1

OAS2

EIF2AK2

HERC6

IFI6

OAS1

USP18

ISG15

CMPK2

DDX58

LY6E

XAF1

IFI35

ACTN4

SPINK5

PARP12

TMEM150B

SAMD 9

HERC5

ODF3B

MTRNR2L6

ANGPTL6

STAT1

LAMP3

SAMD9L

UBE2L6

MX2

TRIM22

Figure 2 MGN1703 significantly modulated the expression of 248 genes in intestinal mononuclear cells, including many genes involved in type I interferon (IFN) responses. (a) Ward link correlation dendrogram for all genes assayed reveals that gene expression patterns cluster with respect to treatment status, rather than by individual (Slatkin-Maddison $P=0.0167$ ). (b) Principal component analysis scores plot for PC1 vs. PC4; the two principal components, which contributed the maximum variance in the analysis. Plots depicting PC2 and PC3 are presented in Supplementary Figure 1. Samples collected at baseline are represented by red circles and samples collected during treatment are represented by blue circles. (c) RNA sequencing (RNASeq) heat map was generated using standardized Z-scores of the genes that were significantly regulated (up or down) in intestinal mononuclear cells after adjusting for a false discovery rate $<0.05$. Individual participants are depicted in the columns and one gene is depicted per row. The dendrogram reveals the relatedness of individual gene expression patterns. A full list of significantly regulated genes can be found in Supplementary Table S3 ( $n=10$ in all graphs). 
T-cell activation (Supplementary Figure 2a), T-cell memory subset proportions (Supplementary Figure $\mathbf{2 b}$ ), or expression of genes known to be involved in T-cell activation (Supple- mentary Figure 2c). Furthermore, there was no change in the number of cell profiles in the intestinal lamina propria positive for myeloperoxidase (Figure 3a and d), an enzyme primarily
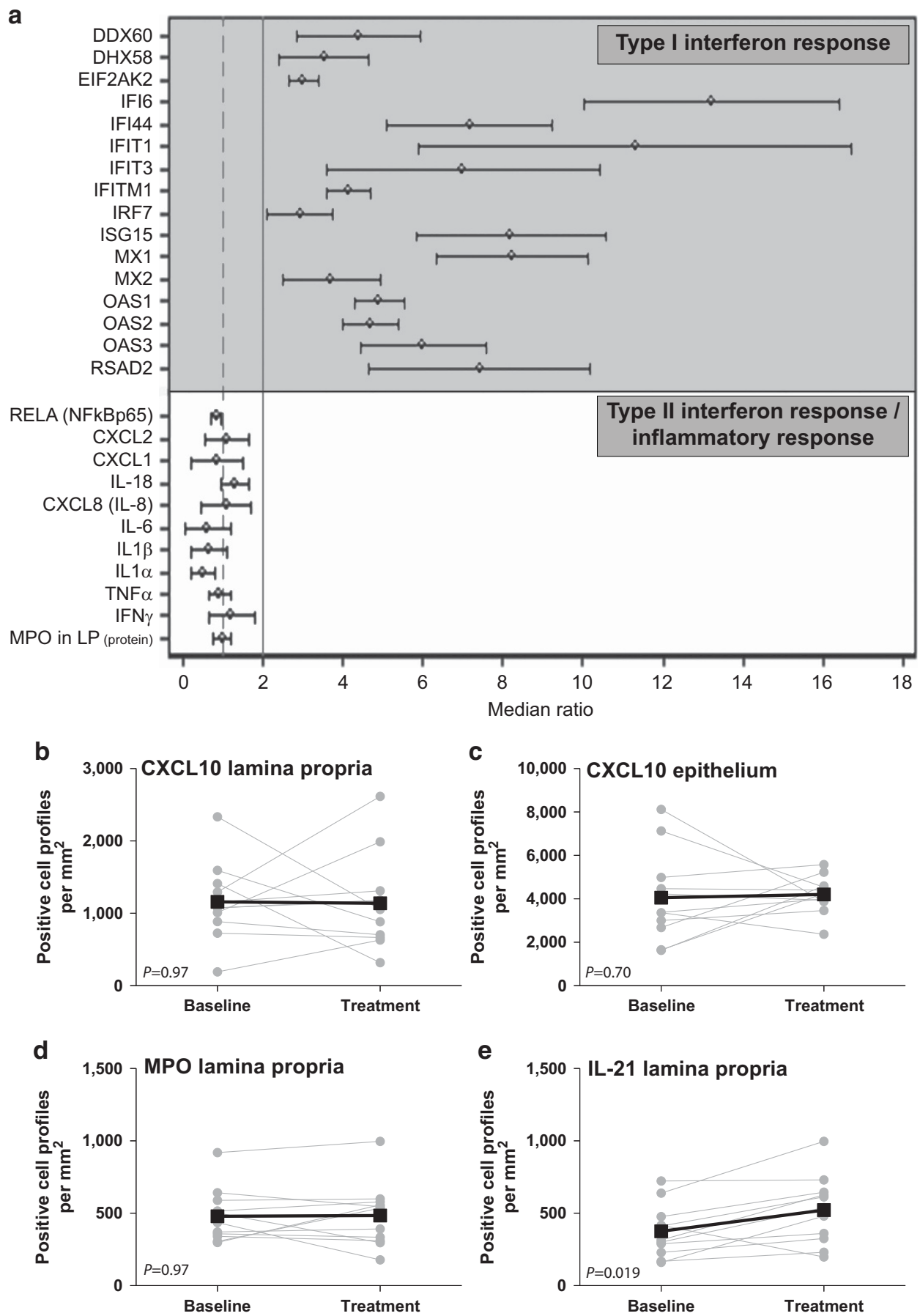

Figure 3 MGN1703 did not induce inflammation in the sigmoid colon. (a) Key type I interferon (IFN) response and inflammatory parameters from the RNA sequencing (RNASeq) were assessed in an equivalence evaluation together with myeloperoxidase data from the immunohistochemistry (IHC) analyses. In this forest plot representation, an upregulation of 2-fold from baseline was set as the threshold for concluding that upregulation had been induced. Confidence intervals in the forest plot are at a $10 \%$ significance level, such that the comparison to the upper equivalence limit is performed at an alpha of 0.05 ( $n=10$ for gene expression parameters and 11 for the myeloperoxidase (MPO) in LP protein parameter). The median of all 16 type I "median ratio" entries is $5.5 \pm 3.0$ s.d. The median of all 11 type II "median ratio" entries is $0.9 \pm 0.3$ s.d. (b-e) Plots show the number of cell profiles expressing CXCL10 (b,c), MPO (d), or IL-21 (e) protein assessed by IHC analyses at baseline and during MGN1703 treatment ( $n=11$ in all graphs). Gray circles indicate results for each individual. Paired data are connected with gray lines. Black squares represent the mean value for all individuals at the indicated time point. Black lines connect longitudinal mean data points. $(\mathbf{b}, \mathbf{d}, \mathbf{e})$ The numbers of positive cell profiles per $\mathrm{mm}^{2}$ within the lamina propria regions are illustrated. (c) The numbers of positive cell profiles per $\mathrm{mm}^{2}$ within the epithelial regions are illustrated. Statistics: Wilcoxon matched-pairs signed-rank test. 
produced by neutrophils ${ }^{33}$ and used as an indicator of general inflammation in the intestinal mucosa. ${ }^{34}$ There was, however, an MGN1703-associated increase in the number of cell profiles in the lamina propria that expressed IL-21 $(P=0.019$, Figure 3e). Together, these data indicate that MGN1703 treatment did not induce a type II IFN response or inflammation in the intestines.

\section{MGN1703 treatment was associated with subtle changes in the intestinal microbiota}

Clinical interventions (e.g., antibiotic treatment for ulcerative colitis) may decrease intestinal inflammation, but often at the expense of reduced microbial diversity in the intestines. ${ }^{35-37}$ Here we performed a comprehensive analysis of the effects of MGN1703 on the gastrointestinal microbiota to determine whether MGN1703 therapy altered microbial diversity. Ribosomal RNA gene amplicon libraries from fecal samples were sequenced and all data sets were rarefied to 14,500 reads for calculations of both $\beta$-and $\alpha$-diversity. No effect of treatment was observed across all fecal samples using analysis of similarity ( $R=-0.105 ; P=0.929 ; 999$ permutations), whereas participant ID was strongly correlated with observed community structure $(R=0.935 ; P=0.001 ; 999$ permutations). This observation was supported by hierarchical clustering of baseline and treatment fecal microbiota structure (Figure 4a). Bray-Curtis similarity values of paired samples (baseline and treatment) ranged from 72.6 to 91.3. Across all pairs of samples no individual taxon, at any taxonomic level, was significantly different between baseline and treatment samples (Figure 4b; Supplementary Table S2). However, small but statistically significant increases between baseline and treatment fecal samples were detected in several $\alpha$-diversity indices, including evenness $(P<0.03$, taxonomic levels of class and order), Shannon index $(P<0.04$, taxonomic levels of class, order, and family), and Simpson's index $(P<0.05$, taxonomic level of family) (Figure $\mathbf{4 c}$ and d, and Supplementary Table S3). The number of bacterial species identified in the fecal samples trended upwards, but did not change significantly with MGN1703 treatment (Figure 4e). Finally, the ratio of Firmicutes-to-Bacteroidetes sequences was slightly, but not significantly, lowered in treatment samples relative to baseline (Figure 4f). These findings indicate that MGN1703 impacts alpha diversity, but had no effect on overall microbial community structure.

\section{In the intestines, baseline TLR9 and IFNAR1 gene expression is inversely proportional to changes in copies of integrated HIV-1 DNA during MGN1703 treatment}

The number of integrated HIV-1 DNA copies reflects the magnitude of the persistent HIV-1 reservoir. ${ }^{38}$ As in peripheral blood CD $4+\mathrm{T}$ cells, ${ }^{30}$ we did not detect significant changes in the copies of HIV-1 DNA in the intestinal mononuclear cells associated with MGN1703 dosing in this cohort $(P=0.23$; Wilcoxon matched-pairs signed-rank test). However, we did observe that some individuals exhibited up to 10 -fold reduction in integrated HIV-1 DNA copies with MGN1703 dosing. To begin elucidating host factors that contribute to the robust decrease in intestinal viral DNA observed in some participants, we analyzed MGN1703-associated changes in copies of integrated HIV-1 DNA together with expression patterns of TLR9, IFNAR1, and IL17RE. The TLR9 gene codes for the target of MGN1703. The IFNAR1 (IFN- $\alpha$ receptor alpha chain) gene codes for a key molecule in the IFN- $\alpha$ receptor. The IL17RE (IL-17 receptor E) gene encodes the IL17C receptor and IL17C is important in innate epithelial immune responses. ${ }^{39-41} \mathrm{We}$ observed that baseline gene expression levels of TLR9 are inversely proportional to the fold-change in integrated HIV-1 DNA copies in the intestines $(P=0.020$; Figure 5a). Furthermore, when the data were stratified according to having a greater than or less than $50 \%$ reduction in integrated HIV-1 DNA copies (stratification point chosen because the data naturally diverged with five participants above and five participants below this value), we observed a significant difference in the number of TLR 9 transcripts present at baseline between the two groups $(P=0.008$; Figure $5 \mathbf{b})$. We also observed a trend for higher baseline levels of IFNAR1 transcripts to associate with greater reductions in integrated HIV-1 DNA $(P=0.087$; Figure $5 \mathbf{c})$ and we detected a significant difference in the baseline IFNAR1 expression when the reduction in integrated HIV-1 DNA copies was $>50 \%$ versus $<50 \%(P=0.024$; Figure $5 \mathbf{d})$. Next, we found that the MGN1703-induced change in IL17RE gene expression is inversely proportional to the fold-change in integrated HIV-1 DNA copies in the intestines $(P=0.035$; Figure 5e). Finally, we observed a significant difference in the MGN1703-induced reduction in IL17RE transcription when the fold-change in integrated HIV-1 DNA copies was $>50 \%$ versus $<50 \%$ $(P=0.032$; Figure 5f $)$. Together, these intestinal data highlight that higher TLR9 and IFNAR1 gene expression before MGN1703 treatment is associated with a greater reduction in integrated HIV-1 DNA levels following treatment.

\section{DISCUSSION}

Here we present the first examination of the in vivo effects of TLR9 agonist therapy in the human colonic sigmoid mucosa and feces. The TLR9 agonist used herein is MGN1703, which is well-tolerated in patients and is currently in phase III testing for the treatment of metastatic colorectal cancer (ClinicalTrials.gov ID: NCT02077868). ${ }^{21}$ Moreover, this drug is being investigated clinically as treatment for small cell lung cancer and melanoma (ClinicalTrials.gov ID number: NCT02200081 and NCT02668770, respectively). There is also interest in using TLR9 agonists to treat inflammatory bowel diseases-like ulcerative colitis $^{9-12}$ and infectious diseases, including HIV, which cause significant gut pathologies. ${ }^{18}$ Given these clinical possibilities, we performed this study investigating the impact of MGN1703 on intestinal homeostasis and HIV persistence in HIV-positive individuals on ART. Although untreated HIV infection is associated with disrupted barrier function and intestinal pathologies, ${ }^{42-44}$ the immune function restoration that occurs during ART ${ }^{45,46}$ supports the generalization of our findings to other indications including malignancy and inflammatory bowel diseases. 
a
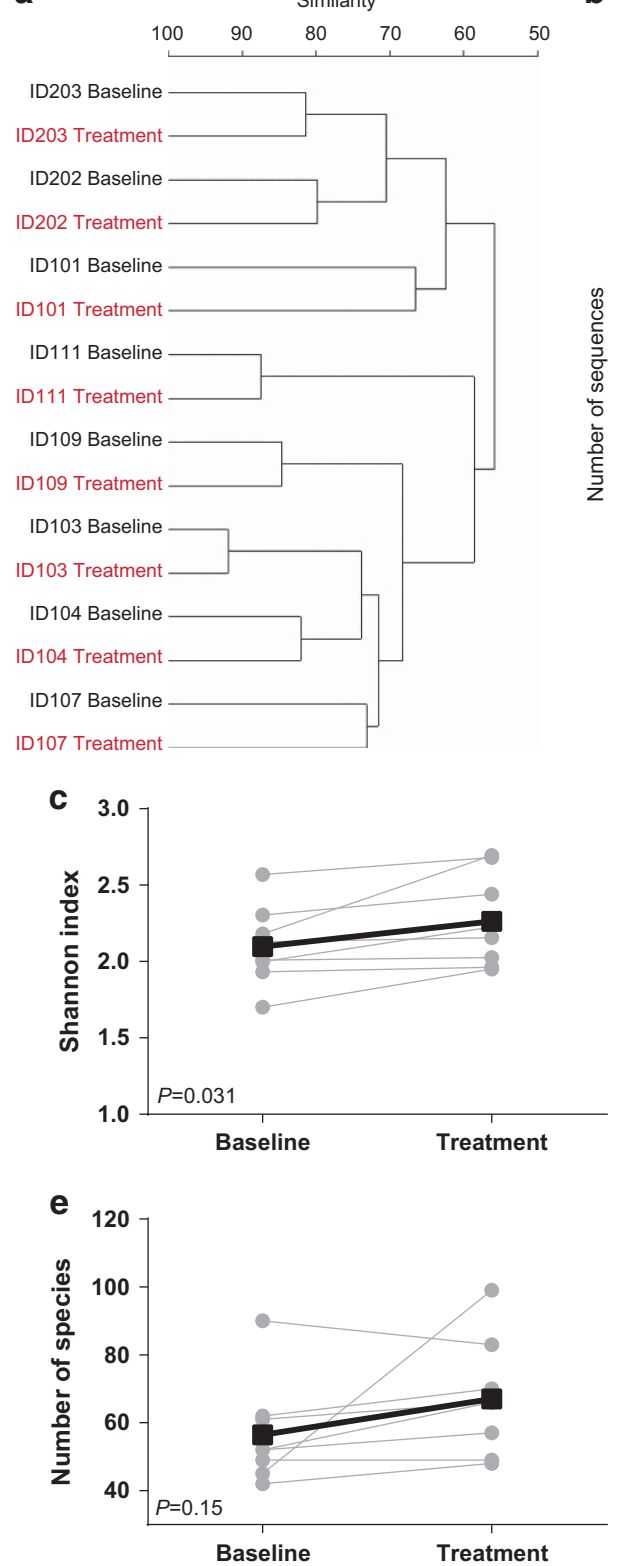

b
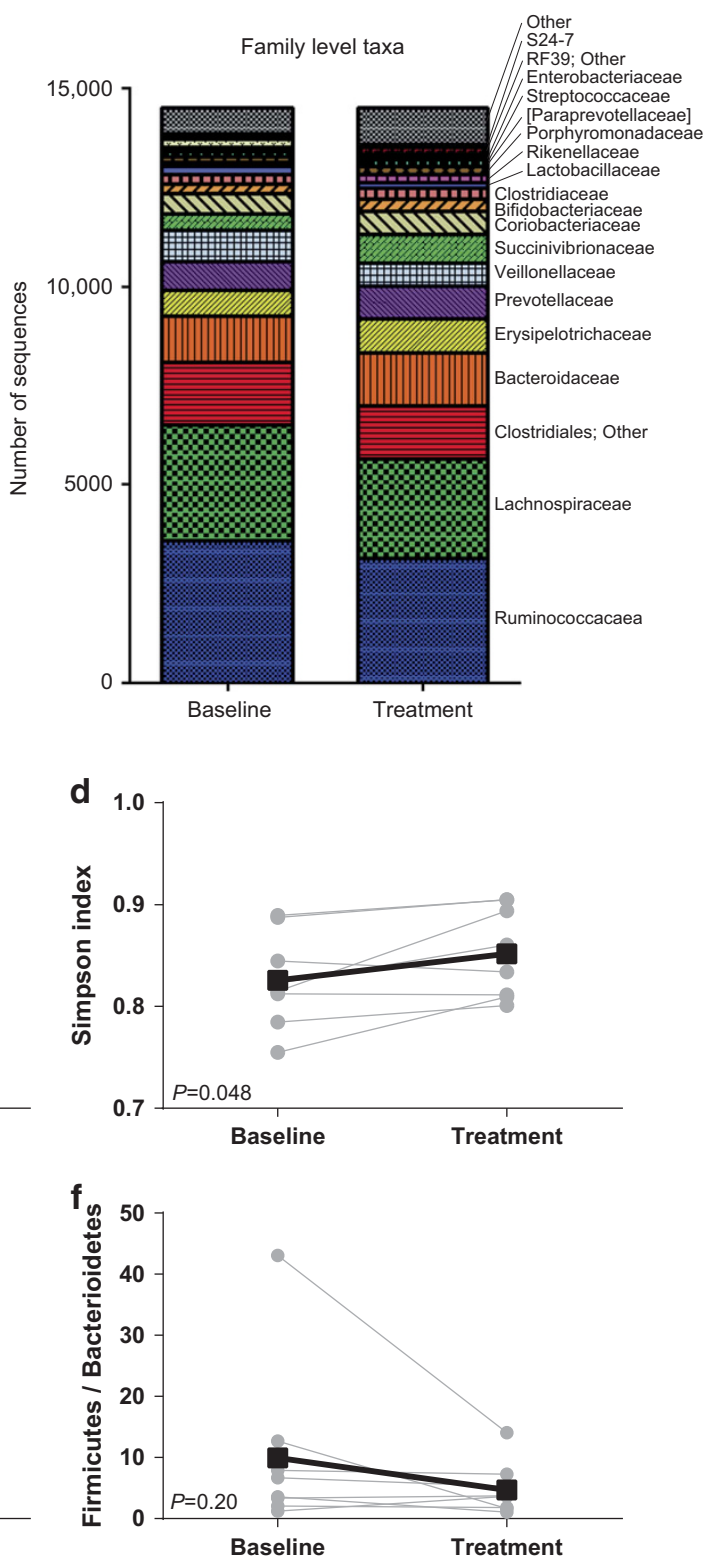

Figure 4 MGN1703 treatment did not radically alter the intestinal flora. (a) Group average dendrogram illustrates relationships between samples at the taxonomic level of family. Individual participants clustered at baseline and treatment with regard to diversity in their microbiota compositions. (b) The rarefied number of sequences $(14,500)$ of microbial taxa (family) at baseline and post-treatment is shown. (c-f) Plots show the Shannon diversity (family) index values (c), the Simpson diversity (family) index values (d), the number of bacterial species identified (e), and the fermicutes over bacterioides ratios (f) at baseline and during MGN1703 treatment ( $n=8$ in all graphs). Gray circles indicate results for each individual. Paired data are connected with gray lines. Black squares represent the mean value for all individuals at the indicated time point. Black lines connect longitudinal mean data points. Statistics for $(\mathbf{c}-\mathbf{f})$ : paired $t$-tests.

MGN1703 activates plasmacytoid dendritic cell to secrete IFN- $\alpha^{19}$ which has a critical role in the immune response to viral infections by inducing the production of proteins with described antiviral activity (e.g., MX1 and ISG15). ${ }^{47,48}$ Accordingly, we observed significant increases in cell profiles positive for MX1 and ISG15 in the colon. These protein-based observations were confirmed by gene expression data demonstrating that the MX1 and ISG15 genes were significantly upregulated during MGN1703 therapy. Further evidence of a strong type I IFN response in the intestine during this treatment was the upregulated expression of many additional IFN response genes including: IFIT3, IFIT5, IFI1, IFI44, OAS1, $O A S 2$, and $O A S 3$. In summary, protein and gene expression data confirm that MGN1703 treatment induced a robust type I IFN response in the sigmoid colon of these individuals that may contribute to clearance of the viral reservoir and durable virologic suppression following cessation of ART.

Given the potency of the induced type I IFN response and the known inflammatory properties of TLR agonists, it is conceivable that a type II IFN response and inflammatory cascade 

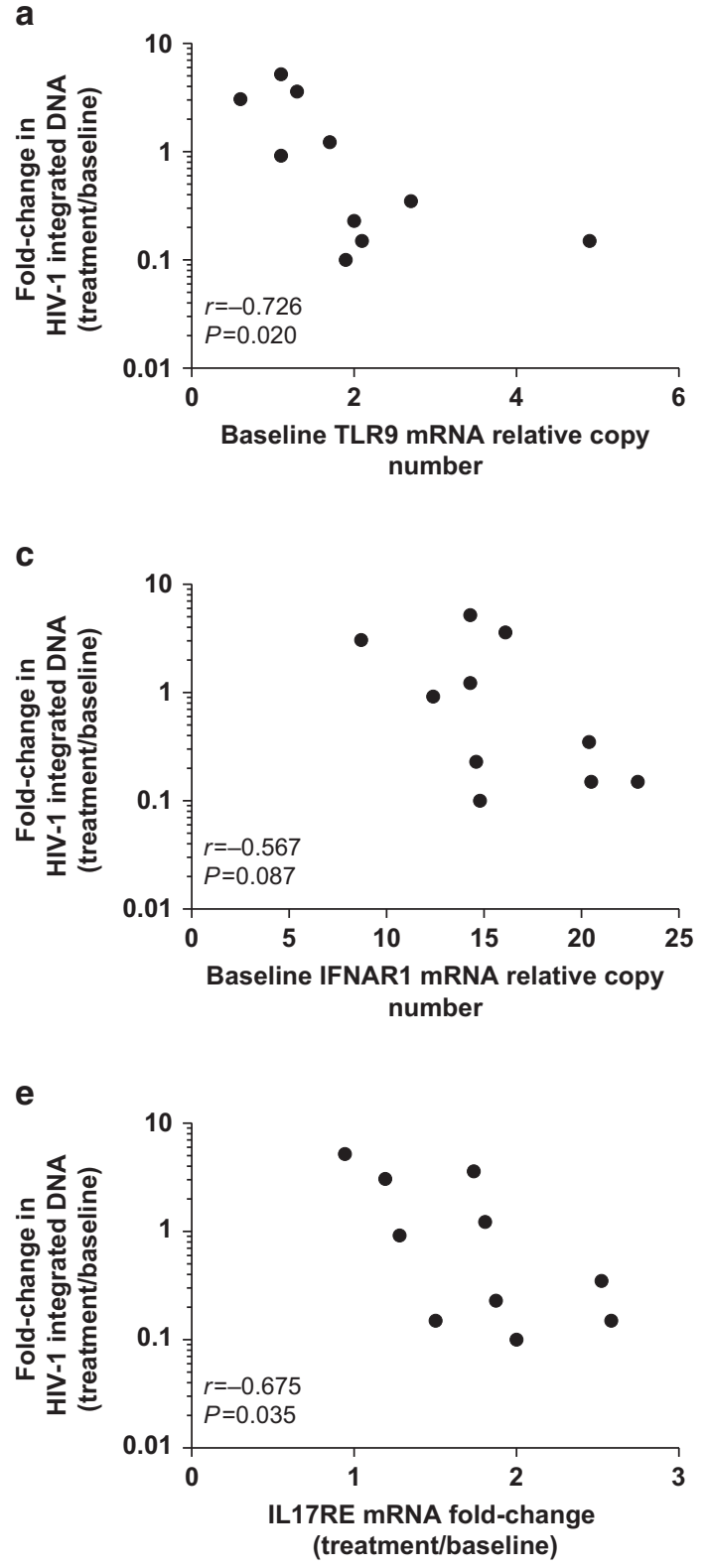

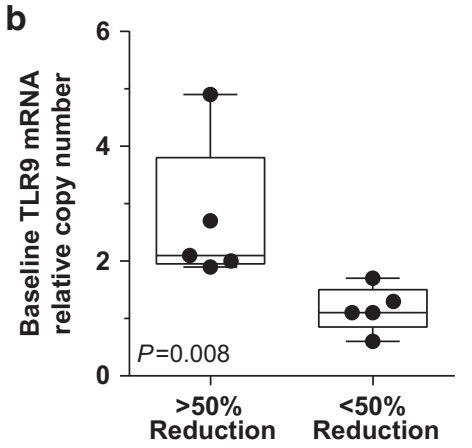

Fold-change in HIV-1 integrated DNA (treatment/baseline)

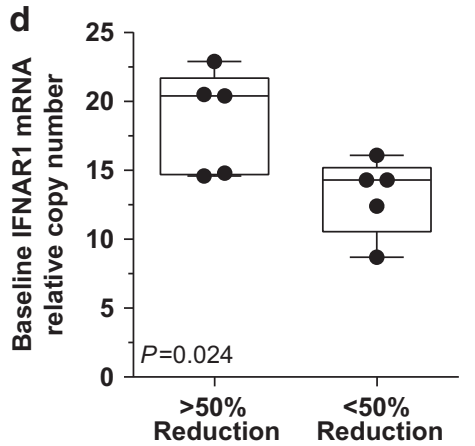

Fold-change in HIV-1 integrated DNA (treatment/baseline)

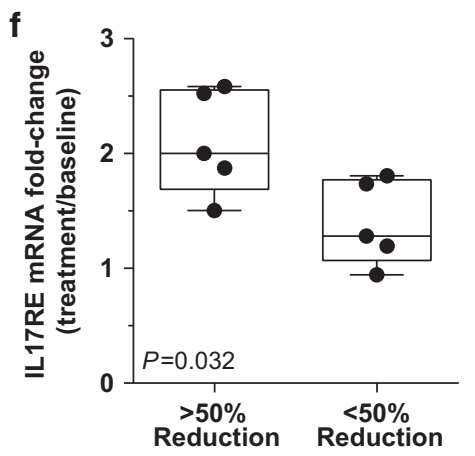

Fold-change in HIV-1 integrated DNA (treatment/baseline)

Figure 5 MGN1703-induced reductions in intestinal mononuclear cell HIV-1 DNA copies is associated with higher baseline TLR9 and IFNAR1 expression. (a,c,e) Bi-variant plots show the fold change in the copies of integrated HIV-1 DNA (left axis) versus the baseline number of TLR9 transcripts (a), the baseline number of IFNAR1 (IFN- $\alpha$ receptor alpha chain) transcripts (c), or the MGN1703-induced fold-change in transcription of IL 17RE (IL-17 receptor $E$ ) (e). Spearman's correlation $r$ and $P$ values are shown. (b,d,f) Data for the baseline number of $T L R 9$ transcripts (b), the baseline number of IFNAR1 transcripts (d), or the MGN1703-induced fold-change in transcription of the IL17RE gene (f) were stratified based on whether the reduction in integrated HIV-1 DNA copies was greater or less than $50 \%$. The HIV-1 DNA values were generated from isolated mononuclear cells. Statistics: Wilcoxon matched-pairs signed-rank test.

could also be triggered by MGN1703. ${ }^{13,14}$ Such an outcome could have a profound negative impact on intestinal homeostasis. Notably, we did not detect any indications of a type II IFN or inflammatory response to MGN1703-either macroscopically during biopsy collection or via the microscopic and genetic analyses employed. The type I IFN cascade can activate monocytes to secrete CXCL10 and induce NK cells to secrete type II IFN (IFN- $\gamma$ ), a potent inducer of CXCL10 production. ${ }^{49}$ Although an increase in CXCL10 protein was observed in plasma during MGN1703 treatment, ${ }^{30}$ we did not find any cohort wide changes in the number of CXCL10-positive cell profiles in the sigmoid colon as would be expected during an inflammatory response. ${ }^{50,51}$ We also quantified the number of infiltrating neutrophils at baseline versus on treatment. In our equivalence evaluation, we found no evidence of inflammatory neutrophil infiltration even though we set a low threshold (twofold increase over baseline) for concluding that inflammation occurred (five- to sevenfold increases in infiltrating 
neutrophils have been reported in animal models of intestinal inflammation). ${ }^{52,53}$ Moreover, the gene expression levels of major inflammatory mediators (e.g., IFNG, RELA, IL6, CXCL8, and $T N F$ ) remained within the same twofold bound. Given that IL-21 combined with ART is known to reduce inflammation in vivo, ${ }^{54}$ the increased number of cell profiles producing IL-21 protein could, at least in part, account for the absence of neutrophil infiltration and inflammation in the intestines during MGN1703 treatment. Together, these observations lead us to conclude that MGN1703 did not induce an inflammatory response that could disrupt intestinal homeostasis.

The stability of the microbiota is another major factor that contributes to the maintenance of intestinal homeostasis. ${ }^{55-57}$ Although HIV-positive individuals have lower microbiota diversity, ${ }^{58}$ there were no major alterations in microbiota diversity associated with MGN1703 treatment. In fact, only subtle increases in multiple diversity parameters were observed. We also observed a nonsignificant trend in this cohort toward a lower firmicutes to bacteroidetes ratio following MGN1703 dosing. Given these microbiota results and our data revealing a lack of MGN1703-associated intestinal inflammation, we conclude that MGN1703 dosing minimally impacts intestinal homeostasis and might lead to modest improvements in the overall gastrointestinal microflora composition.

In the context of a successful HIV eradication strategy, MGN1703 will likely be combined with other interventions. However, it is essential that we have a comprehensive understanding of the individual impact of this drug on HIV-1 reservoirs to fully interpret outcomes in future trials testing combinations of HIV eradication interventions (e.g., to detect synergy). Here we observed that increased transcription of either TLR9 or IFNAR1 at baseline was associated with a reduction in copies of integrated HIV-1 DNA in intestinal mononuclear cells. Thus, a greater number of targets for MGN1703 (i.e., TLR9) and for IFN- $\alpha$ (i.e., IFN- $\alpha$ receptor) at the time of dosing is associated with improved efficacy in eliminating HIV-1 DNA-containing cells in the intestines during the course of treatment. Further downstream in the IFN- $\alpha$ signaling cascade is the action of IL17C through IL-17 receptor E, which leads to antimicrobial peptide production to help maintain intestinal epithelial barrier integrity. ${ }^{39}$ When examining changes in IL17RE gene expression levels following MGN1703 dosing, we found that this parameter was inversely proportional to the reduction in integrated HIV-1 DNA copies in the intestines. These data indicate that each participant's expression levels for key genes directly related to TLR9 function (e.g., TLR and IFN- $\alpha$ receptor alpha chain) substantially impacts the capacity of MGN1703 to reduce the intestinal HIV reservoir.

Potential limitations of our trial include the fact that the study was powered to detect differences in NK cell activation in peripheral blood, ${ }^{30}$ and that the study participants had the option of not consenting to the intestinal biopsy analyses for this sub-study but still take part in the clinical trial. Thus, the secondary endpoint analyses reported here may be unable to detect MGN1703-induced effects. A second potential limitation of this study is the single arm design. However, we established a longitudinal study where each individual's baseline samples served as participant-matched control samples for measuring MGN1703-induced effects. This design accounts for the fact that "normal" human immune parameters vary greatly between individuals but remain relatively stable over time within a given person in the absence of an intervention. ${ }^{59}$ Other potential limitations of the study are that NK cell activation in the intestines was not determined and that gene expression data were obtained using total intestinal mononuclear cell populations. The latter precludes ascertaining cell-type specific expression patterns, but does permit a broader characterization of the impact of MGN1703 in the intestines. The analyses reported here are based upon stool samples and sigmoid colon biopsies and it is possible that MGN1703 effects vary in other regions of the gastrointestinal tract. Thus, future studies characterizing other regions of the gastrointestinal tract are needed. Regarding microbiota analysis, the data reported here are for luminal (stool) microbiota composition and the TLR9 agonist might have a more robust effect on mucosal-associated microbiota, thus future studies need to not only interrogate mucosal-associated microbiota but also assess microbiota function.

In our trial we found that repetitive TLR9 stimulation induced systemic IFN- $\alpha$ production, without causing the severe side effects commonly observed in exogenous pegylated-IFN- $\alpha$ treatment. ${ }^{30}$ Furthermore, the potent type I IFN response, without concomitant inflammation, in the intestines of MGN1703-treated individuals did not perturb intestinal homeostasis but was associated with notable reductions in the tissue viral reservoir in some individuals. Nevertheless, a full understanding of the role(s) of IFN- $\alpha$ in HIV disease and treatment is lacking. ${ }^{60}$ Multiple human and non-human primate studies have associated multiple benefits with exogenous IFN- $\alpha$ treatment of HIV/SIV infection. ${ }^{47,48,61-64}$ More recent reports in humanized mice suggest that blocking the IFN- $\alpha$ receptor during HIV infection could be beneficial; ${ }^{65,66}$ however, this strategy needs to be further explored in HIV-infected patients to fully evaluate such approaches.

In conclusion, we report the in vivo effects of the TLR9 agonist MGN1703 in the human colonic sigmoid mucosa and fecal samples. We demonstrate that intestinal homeostasis was maintained during treatment. These analyses have identified a tissue-specific phenotype whereby MGN1703 treatment did improve immune surveillance via a type I IFN response without inducing inflammation in the sigmoid colon. These data also demonstrate that baseline gene expression levels for TLR9 and IFNAR1 may significantly impact the downstream efficacy of a TLR9 agonist in the intestines. In an HIV treatment context, these findings strongly support the continued development of MGN1703 as an immune surveillance reactivator in HIV eradication approaches. These data also suggest that screening for TLR9 and IFNAR1 expression may aid in predicting drug efficacy regardless of the indication being treated (e.g., malignancies, infections, and inflammatory disorders). 


\section{METHODS}

Study design and participants. Between April and December 2015, we conducted the TEACH study (Toll-like receptor 9 enhancement of antiviral immunity in chronic HIV-1 infection: an investigatorinitiated, single-arm, phase Ib/IIa clinical trial) (ClinicalTrials.gov ID number: NCT02443935). ${ }^{30}$ The study was approved by the National Health Ethics Committee, Denmark (case number 1-10-72-10-15) and the Danish Medical and Health Authorities (case number 2015014125; 7 April 2015) before enrollment. Each participant provided written informed consent before any study procedure. Fifteen HIV-infected individuals (13 males and 2 females) who had received ART for a minimum of 12 months and exhibited $\mathrm{CD} 4{ }^{+} \mathrm{T}$-cell counts above 350 cells per $\mu \mathrm{l}$ and virological suppression $(<50$ copies per $\mathrm{ml}$, at least two measurements per year) received subcutaneous injections of $60 \mathrm{mg}$ MGN1703 (Lefitolimod; Mologen AG, Berlin, Germany) two times per week for 4 weeks, while maintaining ART (Figure 1a). ${ }^{30}$ As part of the study described in (ref. 30), 11 individuals (9 males and 2 females) consented to the optional endoscopic collection of colonic sigmoid biopsies at baseline and during the last week of MGN1703 administration (Figure 1a). The sigmoid biopsies from the fourth dosingweek were collected $24 \mathrm{~h}$ after the last injection of MGN1703. Eight of the 11 intestinal sub-study participants also supplied fecal samples for microbiota analyses at baseline and within 2 weeks of the last study drug dose.

Microbiome analyses. Total DNA was extracted from human fecal samples (FastDNA bead-beating Spin Kit for Soil, MP Biomedicals, Solon, $\mathrm{OH}$ ) collected at baseline and within two weeks of the last study drug dose. Fecal DNA was processed using high-throughput Illumina amplicon sequencing, testing primers described previously ${ }^{67}$ and implementing a modified two-step targeted amplicon sequencing approach. ${ }^{68}$ Negative controls were used with each set of amplifications, which indicated no contamination. Raw sequence data (FASTQ files) were deposited in the National Center for Biotechnology Information Sequence Read Archive under project PRJNA361500. Raw FASTQ files for each sample were processed to merge reads, remove low quality data and chimeras, and perform annotation using the Greengenes 13_8 reference database, as previously described. ${ }^{6}{ }^{6}$ Each sample's sequence set was rarefied to 14,500 sequences $^{70}$ and data were clustered into operational taxonomic units at 97\% similarity. Alpha diversity indices, Bray-Curtis similarity dendograms, and analysis of similarity measurements were calculated within the software package Primer7. ${ }^{71}$ Analysis of similarity was performed at the taxonomic level of family, using square-root transformed data. Differences in relative abundance of individual taxa ( $>1 \%$ of data set), between baseline and treatment samples, were assessed for significance using the Kruskal-Wallis test controlling for false discovery rate, implemented within the software package QIIME. ${ }^{72}$ In SPSS (v.22, IBM, Armonk, NY), paired $t$-tests were used to analyze differences for parametric data satisfying normality test assumptions, via histograms, skewness/kurtosis, and Shaprio-Wilk test; Wilcoxon signed-ranked paired $t$-test was used to analyze non-parametric data. Graphs were created using GraphPad Prism (v5.00, GraphPad Software, La Jolla, CA) software.

Sigmoid biopsies. Sigmoidoscopy was performed as previously described. ${ }^{73}$ In brief, macroscopically normal intestinal tissue samples were taken $\sim 35 \mathrm{~cm}$ from the anal verge. Two biopsies were randomly selected to be fixed in $4 \%$ paraformaldehyde ( $10 \%$ neutral buffered formalin) ( $16 \mathrm{~h}$ at room temperature) and embedded in paraffin blocks. The remaining ( $\sim 16$ total) biopsies were collected in ice-cold Dulbecco's phosphate buffered saline and immediately placed on ice for transportation to the laboratory, where the tissues were immediately digested (enzyme cocktail in RPMI 1640 media: DNase I (1 kunitz unit per $\mathrm{ml}$ ); Elastase (0.064 units per $\mathrm{ml}$ ); (hyaluronidase I-S) (4-10 units per $\mathrm{ml})$; and collagenase II-S $\left(0.25 \mathrm{mg} \mathrm{ml}^{-1}\right)$ ) at $37^{\circ} \mathrm{C}$ for 60 min with constant agitation. Epithelial cells and intraepithelial lymphocytes were not removed prior to digestion to ensure a more complete analysis of the effects of MGN1703 in the intestines. After digestion, intestinal tissue mononuclear cells were collected at the interface of a 40-70\% Percoll density separation centrifugation. Viable cells were counted using trypan blue exclusion and cells were allocated to RNASeq analysis, quantitative PCR for viral DNA, and flow cytometry analyses.

RNASeq analysis of intestinal mononuclear cells. Intestinal tissue mononuclear cells were lysed in RLT Plus Buffer (Allprep isolation kit, Qiagen 80204, Germantown, MD) and lysates were stored at $-80^{\circ} \mathrm{C}$. Total RNA and total DNA were extracted simultaneously from the cell lysates using the Allprep DNA/RNA/miRNA Universal Kit (Qiagen) with on-column DNase treatment (Qiagen RNase-Free DNase Set) during the RNA extraction. RNA samples were quantified using Qubit 2.0 Fluorometer (Life Technologies, Carlsbad, CA) and RNA integrity was checked with 2100 Bioanalyzer (Agilent Technologies, Palo Alto, CA). RNASeq libraries were prepared using the NEBNext Ultra RNA Library Prep Kit for Illumina following manufacturer's recommendations (NEB, Ipswich, MA). Briefly, mRNA was first enriched with Oligo d(T) beads. Enriched mRNAs were fragmented for $15 \mathrm{~min}$ at $94{ }^{\circ} \mathrm{C}$. First-strand and second-strand cDNA were subsequently synthesized. cDNA fragments were end repaired and adenylated at $3^{\prime}$ ends and a universal adapter was ligated to cDNA fragments, followed by index addition and library enrichment with limited cycle PCR. Sequencing libraries were validated using a DNA Chip on the Agilent 2100 Bioanalyzer (Agilent Technologies) and quantified by using Qubit 2.0 Fluorometer (Invitrogen, Carlsbad, CA) and quantitative PCR (Applied Biosystems, Carlsbad, CA).

The sequencing libraries were multiplexed and clustered onto a flowcell. After clustering, the flowcell was loaded on the Illumina HiSeq 2500 instrument according to manufacturer's instructions. The samples were sequenced using a $1 \times 50 \mathrm{bp}$ Single Read configuration. Raw sequence data files $(21,638$ genes) were deposited in the National Center for Biotechnology Information Sequence Read Archive under project number SRP096929. Image analysis and base calling were conducted by the HiSeq Control Software on the HiSeq 2500 instrument. Raw sequence data (.bcl files) generated from the Illumina HiSeq 2500 were converted into fastq files and de-multiplexed using the Illumina bcl2fastq v 1.8.4 program. One mismatch was allowed for index sequence identification. The sequencing reactions were conducted at GENEWIZ, LLC (South Plainfield, NJ).

Quantification of integrated HIV-1 DNA. Integrated HIV-1 DNA copies were quantified using two-step PCR reactions, as previously described. ${ }^{74}$ Integrated HIV-1 DNA was pre-amplified with two Alu primers and a primer specific for the HIV long terminal repeat region, in addition to primers specific for the $C D 3$ gene to determine cell count. Nested quantitative PCR was then used to amplify HIV and $\mathrm{CD} 3$ sequences from the first round of amplification. Specimens were assayed with up to $500 \mathrm{ng}$ cellular DNA in triplicate reaction wells and copy number was determined by extrapolation against a 5-point standard curve (3-30,000 copies), performed in triplicate using extracted DNA from ACH-2 cells. Given that baseline measurements of integrated HIV-1 DNA differ between individuals and given that baseline measures for each individual represent the control samples in this longitudinal study, integrated HIV-1 DNA data are presented as fold change (treatment per baseline).

Cell surface flow cytometry analyses. For flow cytometric analyses, Fc-receptors were blocked with Human Trustain FcX blocking buffer (Biolegend, San Diego, CA) for $5 \mathrm{~min}$ at room temperature and cells were then stained with CD4 PE-Cy7 (SK3), CD8 PerCP-Cy5.5 (RPAT8), CCR7 FITC (G043H7), CD45RA BV421 (HI100), HLA-DR PE (L243), and CD38 BV605 (HIT2) (all from Biolegend). Antibodies were mixed in BD HorizonTM Brilliant Stain Buffer (BD Biosciences, San Jose, CA). Cells were incubated in the dark for $20 \mathrm{~min}$ at $4{ }^{\circ} \mathrm{C}$, then washed twice in phosphate buffered saline containing $2 \%$ fetal bovine 
serum with centrifugation at $300 \mathrm{~g}$ for $5 \mathrm{~min}$ at $5{ }^{\circ} \mathrm{C}$. All sample data were acquired on a FACSVerse (BD Biosciences) and analyses were performed using FlowJo software (v. 10.0.8r1) (Tree Star, Ashland, OR).

Immunohistochemistry. For IHC, ${ }^{75} 5 \mu \mathrm{m}$ tissue sections were cut, mounted, dewaxed and rehydrated, and then treated as described in Supplementary Table S4. Epitope retrieval $\left(15 \mathrm{~min}\right.$ at $\left.98-100{ }^{\circ} \mathrm{C}\right)$ was followed by blocking (TBS $+0.5 \%$ casein; $10 \mathrm{~min}$ ), rinsing (TBS $+0.05 \%$ Tween-20 (TBS-Tw)) and an incubation with primary antibody (60 min). Samples were washed in TBS-Tw and blocked using $1.5 \% \mathrm{H}_{2} \mathrm{O}_{2}$ in TBS-Tw $(5 \mathrm{~min}$ ) (peroxidase block was not performed for myeloperoxidase-IHC). Horseradish peroxidase-conjugated secondary antibodies (Supplementary Table S4) were detected using ImmPactDAB substrate (Vector Labs, Burlingame, CA). All slides were washed in distilled water, counterstained with hematoxylin, mounted with Clear Mount (Invitrogen), dried for $60 \mathrm{~min}$ at $40^{\circ} \mathrm{C}$, and mounted with Permount (Electron Microscopy Sciences, Hatfield, PA).

Quantification of IHC signal. A cell profile was counted as positive on blinded sections via visual inspection (magnification $=\times 200$ ) when IHC signal was associated with a nucleus and when the nucleus was sampled by randomly selected, non-overlaying two-dimensional counting frame $\left(25.600 \mu \mathrm{m}^{2}\right) .^{73}$ This was performed using compound light microscopy with a robotic stage that randomly selected non-overlaying areas for quantification (Visiopharm newCAST, version 5.0.3.1247, Visiopharm, Hoersholm, Denmark). Sections representing the same individual and time point were cut from two separate blocks (biological replicates) and the average of the counted numbers in these replicates are the values presented in the figures. Validation of the IHC quantification strategy was performed according to standard methods before unblinding (Supplementary Table S5). ${ }^{73}$ Quantitative IHC data are stratified into positive cell profiles per $\mathrm{mm}^{2}$ lamina propria or epithelium (insufficient events for IL-21 and myeloperoxidase in the epithelium precluded conclusions for these parameters). Paraffin embedding and preparation cause tissue shrinkage. ${ }^{76,77}$ Thus, the area measurements reported here will not be the same as the true parameters present in vivo.

Statistics. Unless noted, all statistical tests were performed using an alpha of 0.05 . Specific statistical tests (e.g., Spearman's correlation) are indicated in the appropriate figure legend or table footnote. Equivalence evaluation and forest plot generation were performed using R statistical package. ${ }^{78}$ For RNASeq analyses, RNAseq reads were mapped using Tophat to the reference genome hg19. ${ }^{79,80}$ Gene expression quantification in fragments per kilobase of transcript per million mapped reads was calculated using Cufflinks suite including Cufflinks, Cuffmerge, Cuffquant, and Cuffnorm. ${ }^{81,82}$ False discovery rates were computed using the Benjamini-Hochberg procedure to adjust for multiple comparisons in the RNAseq data. ${ }^{83}$ The clustering dendrogram depicting relatedness between gene expression profiles was generated using hierarchical clustering with complete linkage using R. The maximum number of unique tree permutations $(184,756)$ were generated to determine statistical significance, applying a standard $P<0.05$ cutoff (significance was achieved when less than 5\% of randomly generated trees exhibited the same or greater level of segregation between treatment groups than the clustering dendrogram-depicted relatedness in actual expression data). The SlatkinMaddison test was used to evaluate MGN1703 treatment-associated segregation in gene expression patterns (HyPhy_v2.2.4). Principal component analysis was performed using $\mathrm{R}$ on gene expression data for all 21,638 genes assessed. Each gene across samples was standardized with zero mean and unit variance.

SUPPLEMENTARY MATERIAL is linked to the online version of the paper at http://www.nature.com/mi

\section{ACKNOWLEDGMENTS}

We thank the trial participants. Without their efforts, this study would not have been possible. We thank Lene Svinth Jøhnke for her technical assistance. We also thank Dr Jacob D. Estes from the Frederick National Laboratory for Cancer Research for IHC protocols and expert advice. This study was funded by amfAR (grant number 109118), the Danish Council for Independent Research (DFF grant number 5053-00090), Aarhus University Research Foundation (AUFF-E-2016-FLS-8-9), Aarhus University, Denmark, and the NIH-National Institute of General Medical Sciences (R01GM117901). Mologen AG provided the study drug. Standards for quantitative PCR methods for tissue integrated HIV-1 DNA were made available with help from University of California San Francisco-Gladstone Institute of Virology and Immunology Center for AIDS Research (CFAR), an $\mathrm{NIH}$-funded program (P30 Al027763). Centre for Stochastic Geometry and Advanced Bioimaging is supported by Villum Foundation. The funders had no role in the study design, data analyses, data interpretation or writing of the article.

\section{AUTHOR CONTRIBUTIONS}

Concept development: P.W.D., O.S.S., M.T., L.V., M.H.S., L.Ø., and T.A.R. Clinical study and collection of clinical samples: L.V., T.B., A.D., J.A., and A.M.P. Performed laboratory assays/experiments including data analyses and interpretation of the results: A.R.K., M.A.M., M.H.S., L.V., P.A.E., A.D., B.W., M.S., S.J.G., A.N., A.K., X.D., R.O., J.A., J.R.N., A.L., O.S.S., S.K.P., M.T., and P.W.D. First draft of the manuscript: A.R.K. and P.W.D. Critically revising the manuscript for important intellectual content: all authors.

\section{DISCLOSURE}

The authors of this study, except B.W., declare no conflicts of interests. B.W. has intellectual property on MGN1703, receives funding from Mologen AG, and holds shares. M.S. is an employee of Mologen AG.

(c) 2018 Society for Mucosal Immunology

\section{REFERENCES}

1. Hemmi, H. et al. A Toll-like receptor recognizes bacterial DNA. Nature 408, 740-745 (2000)

2. Takeda, K., Kaisho, T. \& Akira, S. Toll-like receptors. Annu. Rev. Immunol. 21, 335-376 (2003)

3. Iwasaki, A. \& Medzhitov, R. Toll-like receptor control of the adaptive immune responses. Nat. Immunol. 5, 987-995 (2004).

4. McClure, R. \& Massari, P. TLR-dependent human mucosal epithelial cell responses to microbial pathogens. Front. Immunol. 5, 386 (2014).

5. Hayashi, K., Sasai, M. \& Iwasaki, A. Toll-like receptor 9 trafficking and signaling for type I interferons requires PIKfyve activity. Int. Immunol. 27, 435-445 (2015).

6. Sasai, M., Linehan, M.M. \& Iwasaki, A. Bifurcation of Toll-like receptor 9 signaling by adaptor protein 3. Science 329, 1530-1534 (2010).

7. Colonna, M., Trinchieri, G. \& Liu, Y.J. Plasmacytoid dendritic cells in immunity. Nat. Immunol. 5, 1219-1226 (2004).

8. Cho, H. \& Kelsall, B.L. The role of type I interferons in intestinal infection, homeostasis, and inflammation. Immunol. Rev. 260, 145-167 (2014).

9. Mannon, P.J. et al. Suppression of inflammation in ulcerative colitis by interferon-beta-1a is accompanied by inhibition of IL-13 production. Gut 60, 449-455 (2011).

10. Furi, I. et al. Epithelial Toll-like receptor 9 signaling in colorectal inflammation and cancer: clinico-pathogenic aspects. World J. Gastroenterol. 19, 4119-4126 (2013).

11. Neurath, M.F. New targets for mucosal healing and therapy in inflammatory bowel diseases. Mucosal Immunol. 7, 6-19 (2014).

12. Wang, Y. et al. Type I interferons for induction of remission in ulcerative colitis. Cochrane Database Syst. Rev. 9, CD006790 (2015).

13. O'Neill, L.A., Bryant, C.E. \& Doyle, S.L. Therapeutic targeting of Toll-like receptors for infectious and inflammatory diseases and cancer. Pharmacol. Rev. 61, 177-197 (2009).

14. Krieg, A.M. Development of TLR9 agonists for cancer therapy. J. Clin. Invest. 117, 1184-1194 (2007).

15. O'Hara, J.R., Feener, T.D., Fischer, C.D. \& Buret, A.G. Campylobacter jejuni disrupts protective Toll-like receptor 9 signaling in colonic epithelial 
cells and increases the severity of dextran sulfate sodium-induced colitis in mice. Infect. Immun. 80, 1563-1571 (2012).

16. Booth, J., Jimbo, S. \& Mutwiri, G. Novel B regs down-regulate TLR9-induced cytokine responses in sheep Peyer's patches. Vet. Immunol. Immunopathol. 148, 157-160 (2012).

17. Byadgi, O. et al. The effect of TLR9 agonist CpG oligodeoxynucleotides on the intestinal immune response of cobia (Rachycentron canadum). J. Immunol. Res. 2014, 273284 (2014).

18. Offersen, R. etal. A novel Toll-like receptor-9 agonist, MGN1703, enhances HIV-1 transcription and NK cell-mediated inhibition of HIV-1 infected autologous CD4 + T-cells. J. Virol. 90, 4441-4453 (2016).

19. Kapp, K., Kleuss, C., Schroff, M. \& Wittig, B. Genuine immunomodulation with dSLIM. Mol. Ther. Nucleic Acids 3, e170 (2014).

20. Schmidt, M., Hagner, N., Marco, A., Konig-Merediz, S.A., Schroff, M. \& Wittig, B. Design and structural requirements of the potent and safe TLR-9 agonistic immunomodulator MGN1703. Nucleic Acid Ther. 25, 130-140 (2015).

21. Wittig, B., Schmidt, M., Scheithauer, W. \& Schmoll, H.J. MGN1703, an immunomodulator and Toll-like receptor-9 agonist: from bench to bedside. Crit. Rev. Oncol./Hematol. 94, 31-44 (2015).

22. Schmoll, H.J. et al. Maintenance treatment with the immunomodulator MGN1703, a Toll-like receptor 9S(TLR9) agonist, in patients with metastatic colorectal carcinoma and disease control after chemotherapy: a randomised, double-blind, placebo-controlled trial. J. Cancer Res. Clin. Oncol. 140, 1615-1624 (2014).

23. Rasmussen, T.A., Tolstrup, M. \& Sogaard, O.S. Reversal of latency as part of a cure for HIV-1. Trends Microbiol. 24, 90-97 (2016).

24. Siliciano, J.D. \& Siliciano, R.F. The latent reservoir for HIV-1 in resting CD4 + T-cells: a barrier to cure. Curr. Opin. HIV AIDS 1, 121-128 (2006).

25. Yukl, S.A. et al. The distribution of HIV DNA and RNA in cell subsets differs in gut and blood of HIV-positive patients on ART: implications for viral persistence. J. Infect. Dis. 208, 1212-1220 (2013).

26. Chun, T.W. et al. Persistence of HIV in gut-associated lymphoid tissue despite long-term antiretroviral therapy. J. Infect. Dis. 197 (March 1), 714-720 (2008).

27. Shan, L. et al. Stimulation of HIV-1-specific cytolytic T lymphocytes facilitates elimination of latent viral reservoir after virus reactivation. Immunity 36, 491-501 (2012).

28. Martinson, J.A. et al. Impact of class A, B and C CpG-oligodeoxynucleotides on in vitro activation of innate immune cells in human immunodeficiency virus-1 infected individuals. Immunology 120, 526535 (2007).

29. Sogaard, O.S. et al. Improving the immunogenicity of pneumococcal conjugate vaccine in HIV-infected adults with a Toll-like receptor 9 agonist adjuvant: a randomized, controlled trial. Clin. Infect. Dis. 51, 42-50 (2010).

30. Vibholm, L. et al. Short-course TLR9 agonist treatment impacts innate immunity and plasma viremia in individuals with HIV infection. Clin. Infect. Dis. 64, 1686-1695 (2017).

31. Sadler, A.J. \& Williams, B.R. Interferon-inducible antiviral effectors. Nat. Rev. Immunol. 8, 559-568 (2008).

32. Nguyen, K.B., Cousens, L.P., Doughty, L.A., Pien, G.C., Durbin, J.E. \& Biron, C.A. Interferon alpha/beta-mediated inhibition and promotion of interferon gamma: STAT1 resolves a paradox. Nat. Immunol. 1, 70-76 (2000).

33. Klebanoff, S.J. Myeloperoxidase: friend and foe. J. Leukoc. Biol. 77, 598625 (2005).

34. Estes, J.D. et al. Damaged intestinal epithelial integrity linked to microbial translocation in pathogenic simian immunodeficiency virus infections. PLoS Pathog. 6, e1001052 (2010).

35. Sartor, R.B. Therapeutic manipulation of the enteric microflora in inflammatory bowel diseases: antibiotics, probiotics, and prebiotics. Gastroenterology 126, 1620-1633 (2004).

36. Dethlefsen, L. \& Relman, D.A. Incomplete recovery and individualized responses of the human distal gut microbiota to repeated antibiotic perturbation. Proc. Natl Acad. Sci. USA 108 (Suppl 1), 4554-4561 (2011).

37. Dethlefsen, L., Huse, S., Sogin, M.L. \& Relman, D.A. The pervasive effects of an antibiotic on the human gut microbiota, as revealed by deep 16S rRNA sequencing. PLoS Biol. 6, e280 (2008).

38. Eriksson, S. et al. Comparative analysis of measures of viral reservoirs in HIV-1 eradication studies. PLoS Pathog. 9, e1003174 (2013).
39. Song, X. et al. IL-17RE is the functional receptor for IL-17C and mediates mucosal immunity to infection with intestinal pathogens. Nat. Immunol. 12, 1151-1158 (2011).

40. Ramirez-Carrozzi, V. et al. IL-17C regulates the innate immune function of epithelial cells in an autocrine manner. Nat. Immunol. 12, 1159-1166 (2011).

41. Chang, S.H., Reynolds, J.M., Pappu, B.P., Chen, G., Martinez, G.J. \& Dong, C. Interleukin-17C promotes Th17 cell responses and autoimmune disease via interleukin-17 receptor E. Immunity 35, 611-621 (2011).

42. Brenchley, J.M. et al. Microbial translocation is a cause of systemic immune activation in chronic HIV infection. Nat. Med. 12, 1365-1371 (2006).

43. Guadalupe, M. et al. Severe CD4 + T-cell depletion in gut lymphoid tissue during primary human immunodeficiency virus type 1 infection and substantial delay in restoration following highly active antiretroviral therapy. J. Virol. 77, 11708-11717 (2003).

44. Estes, J. et al. Collagen deposition limits immune reconstitution in the gut. J. Infect. Dis. 198, 456-464 (2008).

45. Sempowski, G.D. \& Haynes, B.F. Immune reconstitution in patients with HIV infection. Annu. Rev. Med. 53, 269-284 (2002).

46. Macal, M. et al. Effective CD4 + T-cell restoration in gut-associated lymphoid tissue of HIV-infected patients is associated with enhanced Th17 cells and polyfunctional HIV-specific T-cell responses. Mucosal Immunol. 1, 475-488 (2008).

47. Abdel-Mohsen, M. et al. Effects of alpha interferon treatment on intrinsic anti-HIV-1 immunity in vivo. J. Virol. 88, 763-767 (2014).

48. Sandler, N.G. et al. Type I interferon responses in rhesus macaques prevent SIV infection and slow disease progression. Nature 511, 601-605 (2014).

49. Luster, A.D., Unkeless, J.C. \& Ravetch, J.V. Gamma-interferon transcriptionally regulates an early-response gene containing homology to platelet proteins. Nature 315, 672-676 (1985).

50. Mahida, Y.R. The key role of macrophages in the immunopathogenesis of inflammatory bowel disease. Inflamm. Bowel Dis. 6, 21-33 (2000).

51. Mowat, A.M. \& Bain, C.C. Mucosal macrophages in intestinal homeostasis and inflammation. J. Innate Immun. 3, 550-564 (2011).

52. Farooq, S.M. \& Stadnyk, A.W. Neutrophil infiltration of the colon is independent of the FPR1 yet FPR1 deficient mice show differential susceptibilities to acute versus chronic induced colitis. Dig. Dis. Sci. 57, 1802-1812 (2012).

53. Wang, Y. \& Thorlacius, H. Mast cell-derived tumour necrosis factor-alpha mediates macrophage inflammatory protein-2-induced recruitment of neutrophils in mice. Br. J. Pharmacol. 145, 1062-1068 (2005).

54. Micci, L. et al. Interleukin-21 combined with ART reduces inflammation and viral reservoir in SIV-infected macaques. J. Clin. Invest. 125, 4497-4513 (2015).

55. Maloy, K.J. \& Powrie, F. Intestinal homeostasis and its breakdown in inflammatory bowel disease. Nature 474, 298-306 (2011).

56. Sartor, R.B. \& Wu, G.D. Roles for intestinal bacteria, viruses, and fungi in pathogenesis of inflammatory bowel diseases and therapeutic approaches. Gastroenterology 152, 327-339 (2017).

57. Pedron, T. \& Sansonetti, P. Commensals, bacterial pathogens and intestinal inflammation: an intriguing menage a trois. Cell Host Microbe 3, 344-347 (2008).

58. Mutlu, E.A. et al. A compositional look at the human gastrointestinal microbiome and immune activation parameters in HIV infected subjects. PLoS Pathog. 10, e1003829 (2014).

59. Brodin, P. \& Davis, M.M. Human immune system variation. Nat. Rev. Immunol. 17, 21-29 (2017).

60. Deeks, S.G., Odorizzi, P.M. \& Sekaly, R.P. The interferon paradox: can inhibiting an antiviral mechanism advance an HIV cure? J. Clin. Invest. 127, 103-105 (2017)

61. Malim, M.H. \& Bieniasz, P.D. HIV restriction factors and mechanisms of evasion. Cold Spring Harb. Perspect. Med. 2, a006940 (2012).

62. Azzoni, L. et al. Pegylated Interferon alfa-2a monotherapy results in suppression of HIV type 1 replication and decreased cell-associated HIV DNA integration. J. Infect. Dis. 207, 213-222 (2013).

63. Moron-Lopez, S. et al. Short-term treatment with interferon alfa diminishes expression of HIV-1 and reduces CD4 + T-cell activation in patients coinfected with HIV and hepatitis C virus and receiving antiretroviral therapy. J. Infect. Dis. 213, 1008-1012 (2016). 
64. Sun, H. et al. Hepatitis $\mathrm{C}$ therapy with interferon-alpha and ribavirin reduces CD4 T-cell-associated HIV-1 DNA in HIV-1/hepatitis C virus-coinfected patients. J. Infect. Dis. 209, 1315-1320 (2014).

65. Cheng, L. et al. Blocking type I interferon signaling enhances Tcell recovery and reduces HIV-1 reservoirs. J. Clin. Invest. 127, 269-279 (2017).

66. Zhen, A. etal. Targeting type linterferon-mediated activation restores immune function in chronic HIV infection. J. Clin. Invest. 127, 260-268 (2017).

67. Caporaso, J.G. et al. Ultra-high-throughput microbial community analysis on the Illumina HiSeq and MiSeq platforms. ISME J. 6, 1621-1624 (2012).

68. Green, S.J., Venkatramanan, R. \& Naqib, A. Deconstructing the polymerase chain reaction: understanding and correcting bias associated with primer degeneracies and primer-template mismatches. PLOS ONE 10, e0128122 (2015).

69. Bishehsari, F. et al. Light/dark shifting promotes alcohol-induced colon carcinogenesis: possible role of intestinal inflammatory milieu and microbiota. Int. J. Mol. Sci. 17, 1-10 (2016).

70. Gihring, T.M., Green, S.J. \& Schadt, C.W. Massively parallel rRNA gene sequencing exacerbates the potential for biased community diversity comparisons due to variable library sizes. Environ. Microbiol. 14, 285-290 (2012).

71. Clarke, K.R. Non-parametric multivariate analyses of changes in community structure. Aust J Ecol 18, 117-143 (1993).

72. Caporaso, J.G. et al. QIIME allows analysis of high-throughput community sequencing data. Nat. Methods 7, 335-336 (2010).

73. Christensen, A.B. et al. Administration of panobinostat is associated with increased IL-17A mRNA in the intestinal epithelium of HIV-1 patients. Mediators Inflamm. 2015, 120605 (2015).
74. Vandergeeten, C. et al. Cross-clade ultrasensitive PCR-based assays to measure HIV persistence in large-cohort studies. J. Virol. 88, 12385-12396 (2014).

75. Sereti, I., Estes, J.D., Thompson, W.L., Morcock, D.R., Fischl, M.A. \& Croughs, T. et al. Decreases in colonic and systemic inflammation in chronic HIV infection after IL-7 administration. PLoS Pathog. 10, e1003890 (2014).

76. Bruel, A., Christoffersen, T.E. \& Nyengaard, J.R. Growth hormone increases the proliferation of existing cardiac myocytes and the total number of cardiac myocytes in the rat heart. Cardiovasc. Res. 76, 400-408 (2007).

77. Dorph-Petersen, K.A., Nyengaard, J.R. \& Gundersen, H.J. Tissue shrinkage and unbiased stereological estimation of particle number and size. J. Microsc. 204 (Pt 3), 232-246 (2001).

78. R_Core_Team R: A language and environment for statistical computing. R Foundation for Statistical Computing, Vienna, Austria, 2014).

79. Trapnell, C., Pachter, L. \& Salzberg, S.L. TopHat: discovering splice junctions with RNA-Seq. Bioinformatics 25, 1105-1111 (2009).

80. Trapnell, C. et al. Transcript assembly and quantification by RNA-Seq reveals unannotated transcripts and isoform switching during cell differentiation. Nat. Biotechnol. 28, 511-515 (2010).

81. Roberts, A., Pimentel, H., Trapnell, C. \& Pachter, L. Identification of novel transcripts in annotated genomes using RNA-Seq. Bioinformatics 27, 2325-2329 (2011).

82. Trapnell, C. et al. Differential gene and transcript expression analysis of RNA-seq experiments with TopHat and Cufflinks. Nat. Protoc. 7, 562-578 (2012).

83. Benjamini, Y., Drai, D., Elmer, G., Kafkafi, N. \& Golani, I. Controlling the false discovery rate in behavior genetics research. Behav. Brain Res. 125, 279-284 (2001). 